\title{
Auditing Algorithmic Bias on Twitter
}

\author{
Nathan Bartley \\ USC Information Sciences Institute \\ Marina Del Rey, California, USA \\ Emilio Ferrara \\ USC Information Sciences Institute \\ Marina Del Rey, California, USA
}

\author{
Andrés Abeliuk \\ Dept. of Computer Science, University of Chile \\ Santiago, Chile \\ Kristina Lerman \\ USC Information Sciences Institute \\ Marina Del Rey, California, USA
}

\begin{abstract}
Digital media platforms are reshaping our habits, how we access information, and how we interact with others. As a result, algorithms used by platforms, for example, to recommend content, play an increasingly important role in our access to information. Due to practical difficulties of accessing how platforms present content to their users, relatively little is known about how recommendation algorithms affect the information people receive. In this paper we implement a sock-puppet audit, a computational framework to audit black-box social media systems so as to quantify the impact of algorithmic curation on the information people see. We evaluate this framework by conducting a study on Twitter. We demonstrate that Twitter's timeline curation algorithms skew the popularity and novelty of content people see and increase the inequality of their exposure to friends' tweets. Our work provides evidence that algorithmic curation of content systematically distorts the information people see.
\end{abstract}

\section{CCS CONCEPTS}

- General and reference $\rightarrow$ Empirical studies; $\bullet$ Human-centered computing $\rightarrow$ Social media; Empirical studies in collaborative and social computing; $\bullet$ Applied computing;

\section{KEYWORDS}

black-box recommender systems, algorithmic bias, social networks

ACM Reference Format:

Nathan Bartley, Andrés Abeliuk, Emilio Ferrara, and Kristina Lerman. 2021. Auditing Algorithmic Bias on Twitter. In 13th ACM Web Science Conference 2021 (WebSci '21), fune 21-25, 2021, Virtual Event, United Kingdom. ACM, New York, NY, USA, 9 pages. https://doi.org/10.1145/3447535.3462491

\section{INTRODUCTION}

Online social networks (OSNs) have lowered barriers to publication, allowing ever larger numbers of people to share information online and engage in public speech [30]. The downside of unfettered content production is the over-abundance of information, which creates information overload [27]. To mitigate information overload, OSNs curate information for their users, recommending

\section{(c) (†) (?)}

This work is licensed under a Creative Commons Attribution-Share Alike International 4.0 License.

WebSci '21, June 21-25, 2021, Virtual Event, United Kingdom

(c) 2021 Copyright held by the owner/author(s).

ACM ISBN 978-1-4503-8330-1/21/06.

https://doi.org/10.1145/3447535.3462491 a smaller, more tractable subset of content shared by their friends. For instance, OSNs like Twitter, Facebook, Instagram and LinkedIn all create a personalized "social feed"-also known as a timeline or newsfeed-from the content generated by people the users "follow". The social feed mediates users' exposure to information deemed to be sufficiently relevant or interesting by the platform's proprietary curation algorithm. By surfacing the messages the curation algorithm predicts the users will find more interesting, the platforms hope to reduce information overload and improve user experience.

While it is useful for mitigating information overload, the algorithmic curation of content also has downsides. Algorithms may trap users within "filter bubbles" [13] by presenting to them content from like-minded people, limiting the diversity of information to which they are exposed $[8,10]$, and amplifying selective exposure to information [6,34]. Moreover, existing cognitive biases, including social influence [28] and position bias [20], coupled with algorithmic recommendations can amplify online trends, creating "irrational herding" and distorting the perceptions of the underlying value of content $[1,2,11]$.

Despite the growing importance of social media in news consumption, the role of algorithmic curation of content by OSNs has been only partially explored and the findings are conflicting. Prior research has shown that algorithmic recommendations combine with individual decisions to alter the information ecosystem. For example, the introduction of "who to follow" friend recommendation on Twitter, disproportionately accelerated the growth of the already-popular accounts [31]; Google search results of political queries differ significantly based on users' previous browsing history [26]. News stories that have been automatically curated by Apple News come from less diverse sources than the human-curated editorial picks [5]. An experimental study using bots showed how Twitter's environment can steer nonpartisan accounts into partisan echo chambers [9]. However, another study has shown that newsfeed curation can also counteract the self-reinforcing effects of ideological homophily [4] and expose individuals to more diverse viewpoints [21].

Quantifying algorithmic biases of social media curation and recommendation systems is limited by many empirical challenges. We can only observe people's explicit behaviors, e.g., likes or shares of news. Retrieving the information as seen by a user in their social feed cannot be normally done through the API provided by the platform.At best, researchers reconstruct users' timelines by collecting through an API the messages shared by all accounts they follow [16, 27]. Similarly, confounding factors, such as homophily of preferences and social influence, make estimating the effects of algorithmic recommendations hard to disentangle. Compared to 
observational studies, randomized field experiments offer a unique opportunity to address these empirical challenges. In this paper we describe a methodology for auditing a real-world algorithmic curation system using synthetic accounts to run a field experiment. Note that our approach is similar to the "sock-puppet audit" recently described in Sandvig et al., 2014 [29]. We use our methodology to audit Twitter, a popular OSN where many people consume and discuss news [17].

We created matched pairs of user accounts, which we refer to as audit bots or bots for short, both following the same set of accounts, i.e., friends. One account within the pair selects to see personalized content, while the other chooses to see the timeline in reverse chronological order, i.e., without explicit personalization. Importantly, the audit bots only observe and do not perform any actions, such as liking or retweeting posts, or following new accounts.By comparing the personalized timeline to the one presented in (reverse) chronological order and to the unfiltered set of tweets generated by audit bots' friends, we can quantify the biases of algorithmic curation on Twitter. We show that the curated timeline presents a biased sample of friends' tweets, which systematically skews the information users see. We identify three types of bias: popularity, recency and exposure bias. Specifically, we show that while posts observed in the personalized timeline are more popular (receiving more likes and shares at the time of exposure), those in the chronological timeline are newer and more timely. Algorithms also bias exposure to friends, disproportionately amplifying a few of the accounts a user follows.

Contributions. The contributions of this paper can be summarized as follows.

- First, we implement a methodology for measuring the effects of personalization in the exposure of content in social media. We describe how this methodology can be extended to analyze different forms of timeline exposure biases.

- Second, we use this methodology to study personalization on Twitter and show that it systematically skews the popularity and age of tweets people see.

- Third, we use this methodology to study exposure bias on Twitter and demonstrate that personalization distorts a user's view of who the active friends are.

\section{RELATED WORK}

Biases are manifested in digital arenas in many ways and creep in to affect almost all online interactions. Many of the biases on the Internet reflect our own cognitive biases and societal biases and can range from activity biases (e.g., $7 \%$ of people in a sample of Facebook users produced $50 \%$ of the posts in the dataset) to data bias (e.g., the number of links to domains that are in the Global South versus the Global North), and to algorithmic bias [3].

Recommender System Popularity Bias. A number of studies have attempted to quantify the biases of recommender systems, namely popularity bias [23]. In these systems, more popular items tend to be recommended frequently, at the cost of less popular items (which may just as likely be favorable recommendations). Popularity bias shows up across many recommendation systems [18] and multiple domains, for instance in music recommendations [7], algorithmic recommendations reinforce popular artists.

Auditing Exposure Bias. Recent works have attempted to quantify bias in OSNs. Methods that do this tend to fall under one of three categories: 1) simulating simple artificial users and scraping data from the interface directly; 2) asking real users to install a lightweight software extension to scrape the real pages they are exposed to; or 3) simulating more complex users and using the respective API to interact with the service. Sandvig et al., 2014 does a thorough treatment of different methods of blackbox algorithm audit design [29].

Nikolov et al. [22] make use of anonymized web traffic data from Yahoo Toolbar users to analyze two different kinds of biases for web traffic: homogeneity bias, which impacts the number and diversity of information sources you are exposed to, and popularity bias, which selects content from top-ranked sites. They find contradictory evidence to previous work that suggests no significant popularity bias in search engines [14], although this popularity and homogeneity bias is stronger on social media compared to search engines.

Hargreaves et al. [15] propose a transparent and reproducible methodology for auditing an OSN ecosystem's exposure bias that is similar to ours; however, they focus on the Facebook News Feed and its involvement in the 2018 Italian elections. They observe three phenomena: the filtering algorithm tends to select information aligned with a user's perceived political affiliation; the effect is more prominent at the topmost News Feed position; and neutral users are also exposed to non-uniform filtering. They construct several virtual users that follow specific news publishers on Facebook and autoscroll their respective News Feeds to collect impressions, metadata, and the posts themselves at regular intervals throughout the day.

Similar auditing work has been performed recently on Apple News [5]. In this work the authors present an automated method for gathering data from Apple News using an iPhone emulator. They also describe a framework for auditing a news curation platform: an audit should examine the mechanism by which the news is presented, the content, and the end user behavior. They make use of Mechanical Turk workers to analyze the possible personalization the platform performs in practice. They find that the human-curated Top Stories features fewer stories per day and exhibits greater source diversity than the algorithmically-curated Trending Stories.

Facebook researchers themselves have attempted to measure exposure bias for political content on the platform [4]. They observed how 10.1 million U.S. Facebook users interact with shared news on their News Feed. They quantified how much diverse content individuals are exposed to through the algorithmically ranked News Feed, and the links they clicked on. The authors found that a significant portion of partisan users are actually exposed to ideologically cross-cutting content (not symmetrically however), and that individual choices to seek out diverse content seems to play a significant role in what such users are exposed to.

Google Search has also been the subject of similar auditing studies. Robertson et al. [26] recruited participants to complete a survey and install a browser extension that enabled the authors to collect search engine results pages. The authors found little evidence for the "filter bubble" hypothesis when it comes to ideological leanings. 


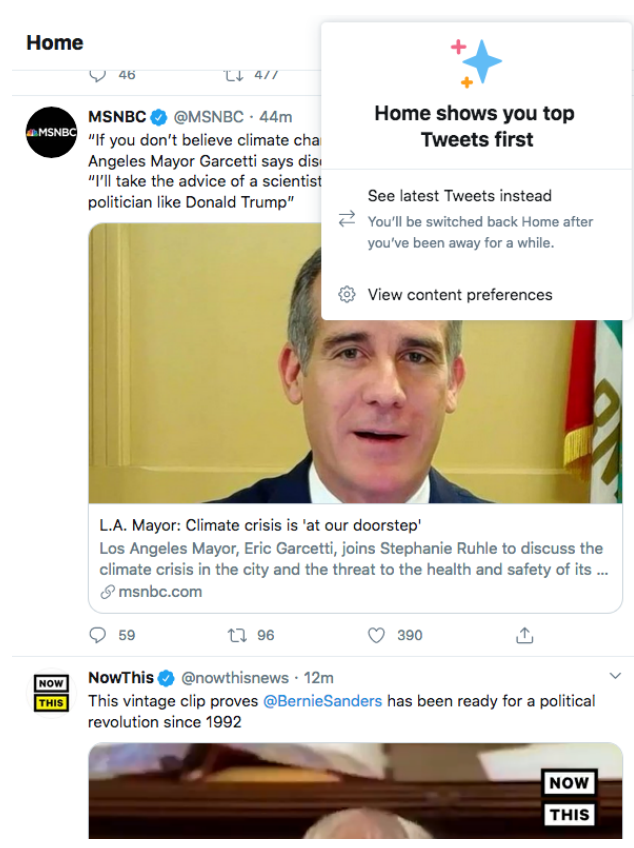

Figure 1: Twitter timeline showing tweets posted by friends. A personalized timeline is shown by default, though the user can choose to see latest tweets, i.e., by reverse chronological order, instead.

A complementary line of work focuses on disentangling the different sources of biases in search engines, specifically, controlling by the bias contained in the data that serves as the input to the algorithmic system [19].

Finally, researchers have worked on simulating partisan users on Twitter and exposing biases in the content they are served in their timelines [9]. They constructed bots with the ability to interact with tweets, generate their own tweets and choose to follow new people or unfollow particular friends. They find conservative accounts are exposed to more low-credibility content, and liberal accounts are exposed to more ideologically moderate content.

\section{METHODS}

In this section, we describe our experimental methodology, including how we created the bots to audit Twitter recommendation algorithms and how we scrape timelines.

\subsection{Experimental Design Overview}

Our experiments measure the impact of Twitter's timeline curation algorithms on the content users see by assessing the differences between the personalized and reverse chronological timelines, or what Twitter refers to as "top tweets" and "latest tweets" respectively, as shown in Fig. 1. We create four matched pairs of bot accounts, who simultaneously log-in several times a day. Each pair is identical, except that one bot in the pair is set up to see tweets in a personalized timeline and the other in reverse chronological order. These bots only observe tweets and do not perform any actions.
While both personalized and reverse chronological accounts experience personalization, this experimental design allows us to isolate the effects of direct algorithmic curation by controlling for other more implicit factors, such as the specific accounts the audit bots follow as well as the time and location (i.e., IP range) from which they $\log$ in. Similarly, by not interacting with any observed tweets, we control for explicit interest signals that may change how the recommender system interacts with each bot.

\subsection{Audit Bots}

Due to scaling issues we encountered in the creation of authenticated accounts, we created a total of eight accounts for four matched pairs of audit bots. Each bot follows one of three sets of accounts, each with roughly sixty accounts sampled from a pool of accounts. Two pairs follow the same set for results consistency. For privacy we only follow public accounts.We refer to accounts that audit bots follow as their friends. A bot's timeline displays posts shared by friends-either original tweets or retweets. Twitter's recommendation algorithm can also surface tweets that are "liked", "retweeted", or replied to by the bot's friends. Similarly, tweets can be surfaced that are posted by the friends of a bot's friend. One bot in each pair selects to see the personalized timeline ("top tweets"), and the other selects the chronological timeline option ("latest tweets") before each session. Our audit bots do not perform any actions, i.e., interacting with tweets nor users. Instead, they log-in six times a day and observe at least 15 tweets per session. These bots used Twitter in May and June 2020.

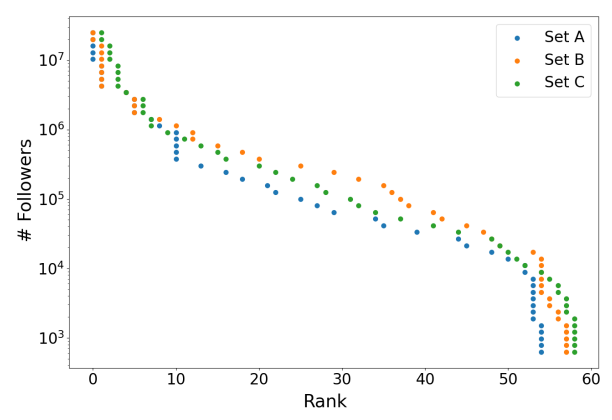

Figure 2: Rank-frequency distribution of the number of followers for each set of friends. Distribution shows large variation in the number of followers.

The eight audit bots and sets of accounts they follow are detailed in Table 1. The accounts followed by each pair of bots were randomly sampled from a curated list of the top 200 most popular anti-science and pro-science Twitter accounts related to the COVID-19 pandemic collected between January 21, 2020 and May 01,2020 [25]. These friend accounts had a large variation in popularity, as shown in Fig. 2, with the number of followers varying between $1 \mathrm{~K}$ and $10 \mathrm{M}$.

\subsection{Timeline Scraping}

Twitter does not facilitate direct scraping of users' timelines through their application programming interface (API). Instead, we use 


\begin{tabular}{c|c|c|c}
$\begin{array}{c}\text { Audit } \\
\text { bot }\end{array}$ & $\begin{array}{c}\text { Friend Set } \\
\text { followed }\end{array}$ & Timeline type & \# Friends \\
\hline per1 & A & Personalized & 55 \\
rev1 & A & Reverse chron. & 55 \\
per2 & A & Personalized & 55 \\
rev2 & A & Reverse chron. & 55 \\
\hline per3 & B & Personalized & 58 \\
rev3 & B & Reverse chron. & 58 \\
\hline per4 & C & Personalized & 59 \\
rev4 & C & Reverse chron. & 59
\end{tabular}

Table 1: Experimental set-up. Each pair of accounts follows the same users, with the number of friends followed adjusted by whether or not the accounts were suspended at the time of writing.

Selenium's Python library to automate access to the timeline for the audit bot accounts we created.

The program works by opening a web browser, logging into the website, scraping the metadata for the tweets observed in the order they appear, and scrolling until a pre-determined number of tweets is observed. We then upload the scraped data to a secure repository. One challenge was that the rendering of the website sometimes resulted in slightly different HTML document structures, which led the scraper to not find the tweet ID. To address missing tweet IDs we relied on the Twitter API to match tweet IDs from the observed user to tweets made at the same time as when the tweet was created. This allowed us to reduce the missing tweet ID rate significantly. We also relied on the Twitter API to give us an accurate count of the number of tweets an account generated for any particular day. However, the endpoint is restricted to the 3,200 most recent tweets, making accurate measurements for very active accounts impractical.

Using this methodology we collected 14,213 tweets across eight different bot accounts until June 23rd, 2020. Of these 14,213 tweets there were 7,929 unique tweets (as defined by tweet ID). The data also contains 1192 missing tweet IDs. After manual inspection, we found the majority of the missing tweets are likely from suspended accounts or are tweets that have been since deleted. This methodology could be used to gather additional information from the timeline, such as "Promoted Tweets", "Who to Follow" recommendations in the sidebar, as well as the presented "Trending Topics."

The rank-frequency distributions of observed tweets and total tweets generated by friends per pair of bot accounts are presented in Fig. 3. This figure shows the number of tweets per account, in decreasing order of frequency (the most active account is ranked 0 , the second most active account is ranked 1, and so on). Each panel represents a set of accounts followed by our bots, and we compared the observed tweets in each algorithmic timeline as well as the total activity produced by the accounts. We highlight two main observations: 1) the observed distribution is heavy tailed; 2) the observed tweets correspond to below $10 \%$ of all the content created by friends during the time frame of the study.

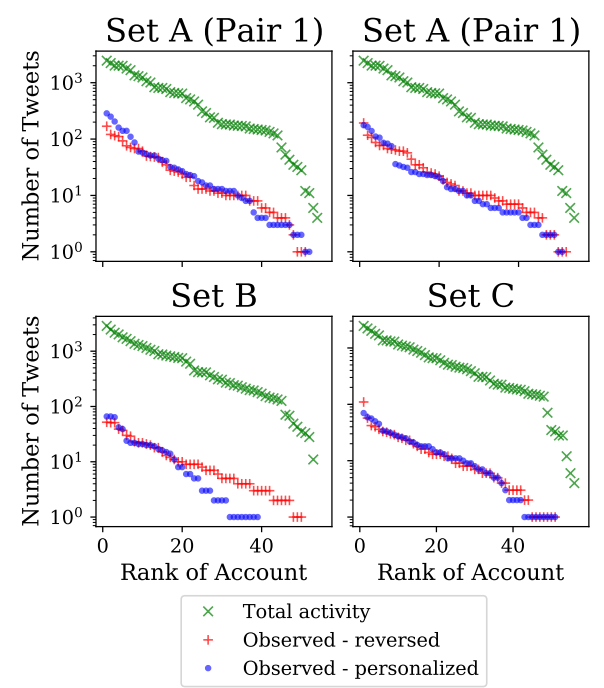

Figure 3: Rank-frequency distribution of observed tweets \& actual activity per set. Green is actual user activity, red is observed user activity in the reversed condition, and blue is observed user activity in the personalized condition. Y-axis is in log scale.

\subsection{Measuring Inequality}

To measure the exposure bias, we rank the friends our audit bots follow by their activity, i.e., the number of tweets they post over a fixed time span. We then compute the Gini coefficient over this observed friend activity. Low value means that all friends post similar number of messages, while a high Gini coefficient means that few friends are responsible for a large share of tweets. The Gini coefficient is defined as follows:

$$
G=\frac{1}{2 * \mathrm{~N}_{u} * \sum_{i} x_{i}} \sum_{i=1}^{\mathrm{N}_{u}} \sum_{j=1}^{\mathrm{N}_{u}}\left|x_{i}-x_{j}\right|
$$

where $x_{i}\left(x_{j}\right)$ is the number of tweets made by friend $i(j)$, and $N_{u}$ is the number of friends of an auditing bot. We repeat this to measure the popularity bias by using the number of likes friends' tweets receive.

\section{RESULTS}

We compare messages shown in the personalized and chronological timelines, and identify systematic differences in their age and popularity. We also show that Twitter's algorithm distorts perceptions of friend activity.

\subsection{Recency Bias}

Figure 4 compares the age of tweets at the time they appeared in the timeline. Tweets shown in the personalized timeline are often much older than those shown in the reverse chronological timeline. The median age of tweets observed in all chronological timelines in our experiments is about 55 minutes. This stands in contrast to the three hour median age of personalized timeline tweets. 


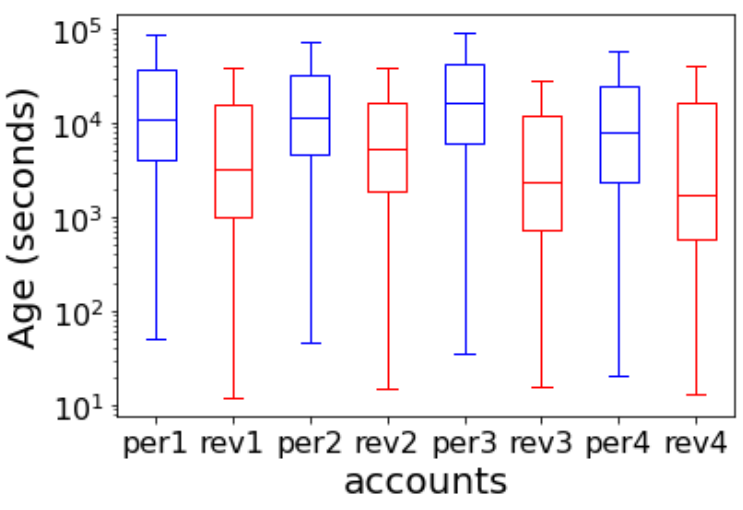

Figure 4: Biases of Twitter personalization algorithm. (a) Tweets in a personalized timeline (per1 through per4) are generally older than the latest tweets shown in the chronologically ordered timeline (rev1 through rev4). Tweet age is measured as the number of seconds between its creation and exposure in the user's timeline. Y-axis is in log scale.

\subsection{Popularity Bias}

Figures 5(a) and 5(b) show that tweets seen in the personalized timeline are more popular than ones observed in the chronological timeline as measured by the number of likes and retweets they received by the time of exposure. Half of the tweets seen in the personalized timelines in our experiment received at least 562 likes and 172 retweets. In comparison, the median popularity of tweets seen in the reverse chronological timelines was 35 likes and 17 retweets. To normalize the effects of age observed in section 4.1, we use Twitter API to retrieve the number of likes all tweets accumulated over two months following their creation, long after their popularity stabilized. We were only able to retrieve the number of likes for 5981 tweets, as some tweets were deleted by owners. Figure 5(c) shows that tweets in the personalized timeline typically have many more likes than those in the reverse chronological timeline.

There are two potential explanations for this observation. The first explanation is that Twitter is very good at picking out interesting tweets to feature in the personalized timeline. These interesting tweets go on to receive many more likes over their lifetime than other tweets. If early popularity is correlated with later popularity, then simply choosing tweets accruing a large number of likes in the early stages will be enough to observe this effect. Alternatively, just being featured in personalized timelines allows these tweets to accrue more likes. We leave this question for future research.

\subsection{Exposure Bias}

By selecting a sample of tweets posted by friends to display in a user's timeline, Twitter subtly alters and distorts a user's perception of who the active friends are. We call this exposure bias. To demonstrate the exposure bias, we compare the actual activity of friends to their observed activity in the personalized and chronological timelines.

4.3.1 Algorithmic Curation Amplifies Inequality. First, friend activity is highly heterogeneous. Already in Fig. 3 we see that some of the

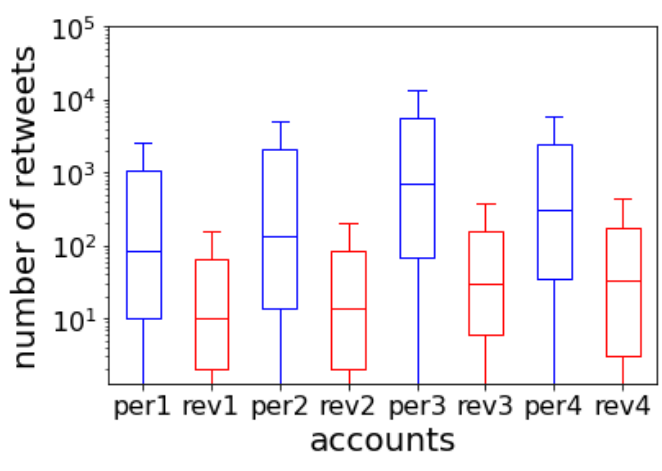

(a) Observed popularity (retweets)

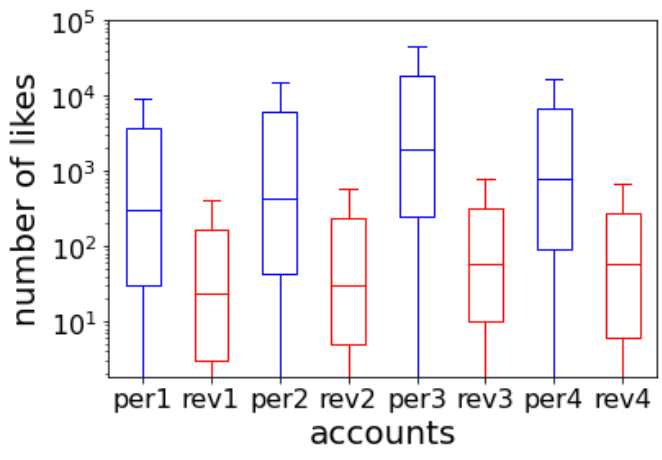

(b) Observed popularity (likes)

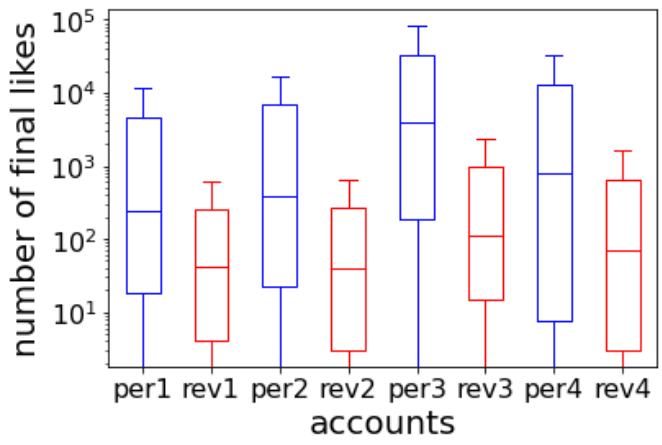

(c) Final popularity (likes)

Figure 5: Popularity bias of Twitter personalization algorithm. Tweets shown in the personalized timeline have more (a) likes and (b) retweets at the time of exposure than tweets in the chronologically ordered timeline. (c) Personalized tweets also accrue more likes over their lifetime than tweets shown in the reverse chronologically ordered timeline. All boxplots are shown without outliers and are presented in $\log$ scale.

friends our audit bots follow are orders of magnitude more active than others. These friends account for the disproportionate share of 


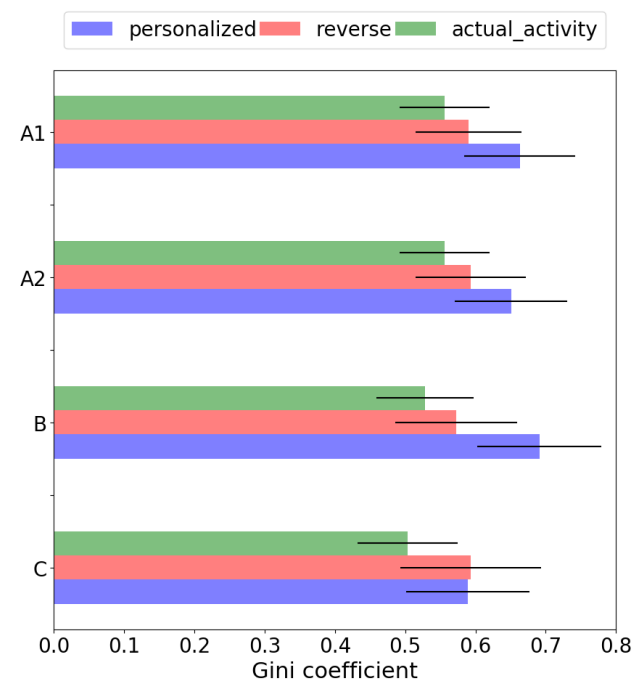

Figure 6: Inequality of exposure to friends' tweets. Gini coefficient of friend activity computed across all sessions shows higher inequality for the observed activity of friends compared to their actual activity. Moreover, inequality of exposure to friends through the personalized timeline is larger than through the reverse chronological timeline. Error bars are 95\% confidence intervals are computed via 1000 bootstrapped samples over sessions.

the overall activity, increasing inequality. To compute the Gini coefficient, we compute the number of tweets that friend posted within the duration of the experiment. Similarly, we compute the number of that friend's tweets that appeared in each bot's timeline. We then bootstrap over 1000 samples of individual sessions and compute the Gini coefficient for each sample for each condition. Figure 6 shows that the inequality of the actual friend activity (green bars) for the four friend sets ranges between 0.522 and 0.580 . In comparison, the Gini coefficient of income inequality in the US, which is perceived to be large, is around 0.4 in recent years. Strikingly, the observed activity of friends in the personalized timeline (Fig. 6, blue bars) is more skewed than their actual activity. Gini coefficient of observed friend activity in the personalized timeline ranges between 0.585 (set C) and 0.703 (set B). Even more surprising, friend activity observed in the chronological timeline (red bars) is also more skewed than their actual activity, ranging from 0.565 (set B) to 0.608 (set A). We ran two-sided t-tests for each pair of the three conditions (personalized, reverse, and actual activity) within each set of friends. We do not report the statistics due to space constraints, however each two-sided test (e.g., $H_{0}: \mu_{\text {pers,A1 }}=\mu_{\text {rev,A1 }}$ ) was significant under $p<0.05$ for each test, suggesting the ordering of the means in Fig. 6 holds.

4.3.2 Algorithmic Curation Distorts Ranking. Another way to demonstrate exposure bias is by ranking users by their actual and observed activity. Entity ranking is widely used on social media platforms to identify most active or most influential users. Previous work has shown how Twitter's API, which returns a real-time sample of tweets, distorts entity ranking for the most active accounts [33].

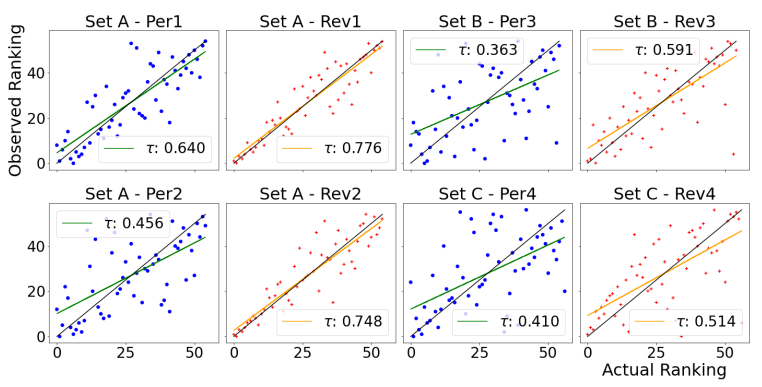

Figure 7: Ranking distortion of algorithmic curation. The plot shows ranking of friends based on the volume of tweets observed in each timeline vs ranking according to the actual volume of friends' tweets. Kendall's $\tau$ coefficient reports correlation for each timeline. Black lines show unbiased association $Y \sim X$. Green and orange lines show best linear fits to demonstrate trend. Friends below the line suggest promotion of the friend in the auditing bot's timeline, and friends above the line suggest demotion.

Here, we show that Twitter's curation algorithm can similarly distort entity ranking and user perceptions of who the active friends are.

To measure rank distortion, we rank friends within each friend set according to the number of their tweets bots observed in the personalized or the reverse chronological timelines. Figure 7 plots these "observed ranking" of friends versus their "actual ranking", based on the volume of tweets these friends generated over the course of the experiment. If actual rankings completely predicted the ranking based on the observed tweets, we would see a diagonal $y=x$ line in the plot. Instead, we see high variance in the rankings. We also observe a consistently higher correlation for the reverse chronological timeline than the personalized timeline, which suggests a higher distortion of entity ranking in the curated tweets. Kendall's $\tau$ for entity ranking using actual tweets and those in the personalized timeline is between 0.36 and 0.64 , and for the reverse chronological timeline it ranges between 0.51 and 0.78 . For comparison, Wu et al. [33] determined that Kendall's $\tau$ for correlation of entity ranking based on sampled and complete tweets is 0.74 . This suggests that timeline curation distorts reality even more than Twitter data sampling does.

4.3.3 Explaining Exposure Bias. How does algorithmic timeline curation amplify the inequality of exposure? Can this inequality be gamed by individual accounts? One hypothesis we explore is that this inequality exposure is the result of the cumulative advantage process, a ubiquitous phenomenon that explains the self-reinforcing inequality pattern in many natural and social systems [24], for example, popularity bias in web traffic due to PageRank-like metrics [22]. This process can be identified by a super-linear relationship between the actual and observed activity, giving a disproportionate share of exposure to already highly active accounts. Figure 8(a) depicts the relationship between the average number of times a friend's tweet is observed versus the number of tweets they have posted during the experiment, for each timeline condition. Actual activity is binned logarithmically to account for the heavy-tailed 


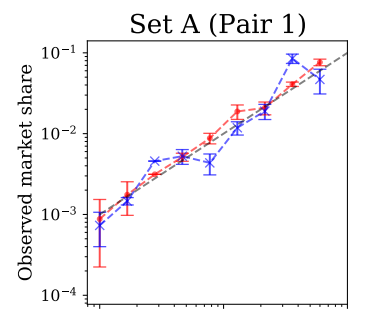

Set B

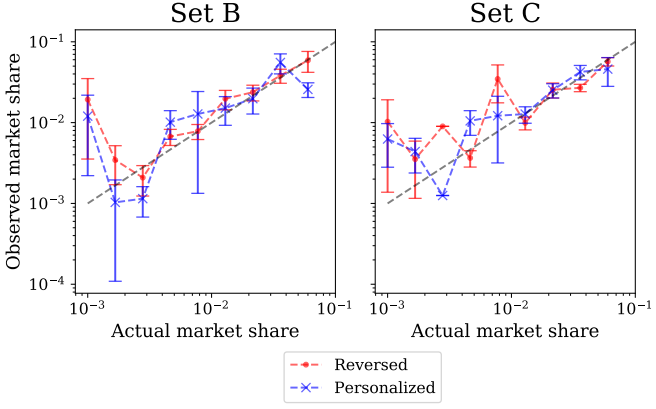

(a) Observed vs Actual Activity

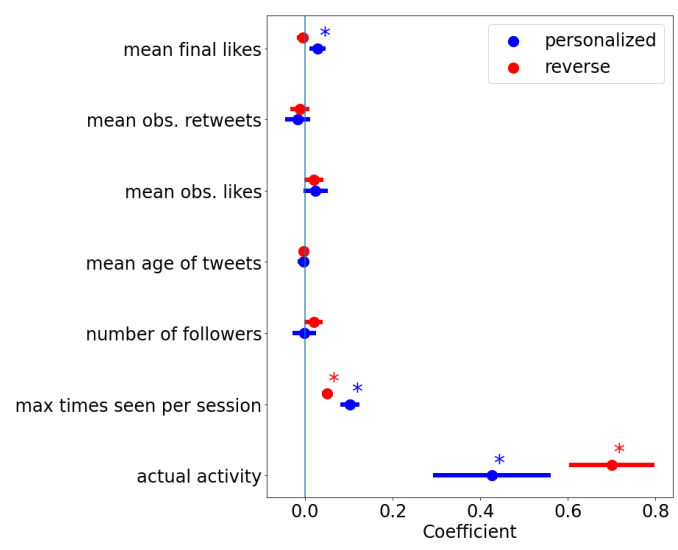

(b) Regression Coefficients

Figure 8: Marketshare analysis. (A) Observed friend activity vs actual activity. Relationship between averaged observed market share activity per account and actual activity market share per account with logarithmic binning. Error bars depict standard error of the mean. Red dots represent the observations in the reverse chronological timeline; blue crosses for the personalized timeline; black dashed line is the identity line. (B) Regression analysis of the observed marketshare of friend activity. The features acting as dependent variables are listed on the left. Asterisks show significant regression values at $p=0.05$ significance level.

distribution (see Fig. 3). We do not observe any systematic deviation from a linear relationship (i.e., having slope one on the log-log scale and depicted with a black dashed line in Fig. 8(a)). Moreover, there does not seem to be a significant difference between the personalized and reverse chronological timelines in this growth rate: the differences between types of accounts seem to be in the variance for more highly active friends. Indeed, the higher levels of inequality exhibited by the personalized timeline is due to a few outliers receiving high exposure.

We also explore factors affecting observed friend activity. We run two separate regressions, with coefficients reported in Fig. 8(b). The regressions predict the outcome-marketshare of observed friend's activity-using features that include marketshare of friend's actual activity, maximum number of times friend's tweets were seen in a session, number of followers of the friend, mean age, likes and retweets of friend's observed tweets, and the mean popularity (final likes) of friend's tweets. The first two features have significant coefficients for both the personalized and reverse chronological timelines. However, the mean number of final likes is significantly positive for the personalized timelines only. This suggests that Twitter selects popular tweets for the personalized timeline, and it is this selection that increases the inequality of exposure to friends in the personalized timeline. Note that actual activity has a significantly larger coefficient for reverse chronological than personalized. Similarly, the regression better explains friends' activity observed in the reverse chronological timelines than the personalized timelines $\left(R^{2}=0.724, R^{2}=0.655\right.$, respectively). This is consistent with Twitter selecting recent tweets for the reverse chronological timeline.

\section{DISCUSSION}

Twitter's timeline curation algorithm subtly changes which friends' tweets the user observes. This is seen both in the inequality of the observed activity and through rank distortion. As a result of curation the user sees a different distribution of activity, with a few of the friends claiming a disproportionate share of the timeline. This is also observed in the ranking: the most active friends observed on the timeline are usually different from who the most active friends actually are. Surprisingly, the chronological timeline also distorts observed friend activity, although not as much.

The lack of significant difference between the two conditions in the slopes of the relationships could be due to the fact that the audit bots did not interact with the tweets, which according to Twitter is one signal used to decide how to rank tweets in a user's timeline [32]. Without interactions, the personalized ranking is unable to use user-specific signals, and thus, more general signals like friend's activity or the number of followers play a stronger role in determining the ranking. On the other hand, lack of significant difference could be due to measurement errors and limited data as well.

The proposed framework has a few limitations. The auditing tool requires regular maintenance, since minor changes in the HTML rendering of the platform can affect the collection of data. Similarly, additional changes to the interface might warrant new structures in the data. For instance, if a friend can control who can reply to their tweets, that may be useful information to maintain in evaluating the relationship of the friend to the user.

Further work includes more faceted analysis based on the content of the tweets. An example of this kind of content analysis can be found in recently published work from the Economist. They did a sentiment analysis on a cloned profile of the 45th President of the United States' Twitter account, demonstrating tweets on 
the algorithmic feed were more emotive than the tweets on the chronological feed [12].

Additional work would also include simple interactions with tweets to account for how the personalization might change over time [8]. This might involve integrating a simple behavior model, similar to Chen et al. (2020), which will either retweet, like, or follow the user who generated the tweet with specific probabilities. This could include allowing the audit bots to develop an orientation that changes over time.

Further work should also be done in simulating a user feed length's impact on inequality. We expect that at least in the reverse chronological timeline, that as the sessions get longer we will eventually observe all of the tweets each friend produces, therefore minimizing exposure inequality. Similar work should also be done to analyze other user-level characteristics like the number of friends each friend has, the number of tweets each friend liked during a time period, and the number of lists each friend is a part of. Our methodology can help gather data to study these open questions.

Ethical Considerations. To be considerate of user privacy in a networked public sphere like Twitter, we changed all the account profiles to identify as research bots and made an explicit effort to follow only public accounts. Since some accounts have since been suspended or otherwise deleted, we also make an effort to remove such accounts (and respective tweets) from analysis.

The study design was classified as Exempt from full review by USC IRB.

Data Disclosure. For replication purposes, we have hosted the tweet IDs and time of exposure at https://bit.ly/3tBhRPw.

\section{CONCLUSION}

Twitter's personalization and curation algorithms that assemble a user's timeline from the tweets posted by the user's friends subtly distort and bias the information users receive. Due to their utility, some of these biases work as expected. Compared to the chronologically ordered timeline that shows friends' latest tweets, the tweets shown in the personalized timeline tend to be older and more popular. Interestingly, the popularity advantage sticks and these tweets end up garnering more likes than tweets that appeared in the chronological timeline only. This has ramifications in the broader scientific literature around OSNs, especially those that rely on timeline reconstruction via the API.

In this paper, we have implemented a sock-puppet audit for measuring the effects of personalization on the exposure to content and friends in social media. We have demonstrated empirically that a fixed-length timeline session is impacted by different biases like exposure bias, popularity bias, and recency bias. Given the reach OSNs have in spreading information, it is important that we understand the mechanisms and interfaces that exist between individuals and the information they are exposed to.

\section{ACKNOWLEDGMENTS}

This work was funded in part by DARPA (under contracts W911NF18-C-0011 and W911NF-17-C-0094) and AFOSR (under contract FA9550-20-1-0224).

\section{REFERENCES}

[1] Andrés Abeliuk, Gerardo Berbeglia, Manuel Cebrian, and Pascal Van Hentenryck. 2015. The benefits of social influence in optimized cultural markets. PloS one 10, 4 (2015), e0121934.

[2] Andrés Abeliuk, Gerardo Berbeglia, Pascal Van Hentenryck, Tad Hogg, and Kristina Lerman. 2017. Taming the unpredictability of cultural markets with social influence. In Proceedings of the 26th International Conference on World Wide Web. International World Wide Web Conferences Steering Committee, 745-754.

[3] Ricardo Baeza-Yates. 2018. Bias on the web. Commun. ACM 61, 6 (2018), 54-61.

[4] Eytan Bakshy, Solomon Messing, and Lada A Adamic. 2015. Exposure to ideologically diverse news and opinion on Facebook. Science 348, 6239 (2015), 1130-1132.

[5] Jack Bandy and Nicholas Diakopoulos. 2020. Auditing news curation systems: A case study examining algorithmic and editorial logic in apple news. In Proceedings of the International AAAI Conference on Web and Social Media, Vol. 14. 36-47.

[6] Michael A Beam. 2014. Automating the news: How personalized news recommender system design choices impact news reception. Communication Research 41, 8 (2014), 1019-1041.

[7] Òscar Celma and Pedro Cano. 2008. From hits to niches? or how popular artists can bias music recommendation and discovery. In Proceedings of the 2 nd KDD Workshop on Large-Scale Recommender Systems and the Netflix Prize Competition. 1-8.

[8] Allison JB Chaney, Brandon M Stewart, and Barbara E Engelhardt. 2018. How algorithmic confounding in recommendation systems increases homogeneity and decreases utility. In Proceedings of the 12th ACM Conference on Recommender Systems. ACM, 224-232.

[9] Wen Chen, Diogo Pacheco, Kai-Cheng Yang, and Filippo Menczer. 2020. Neutral Bots Reveal Political Bias on Social Media. arXiv preprint arXiv:2005.08141 (2020).

[10] Sunshine Chong and Andrés Abeliuk. 2019. Quantifying the Effects of Recommendation Systems. In 2019 IEEE International Conference on Big Data (Big Data). IEEE, 3008-3015.

[11] Giovanni Luca Ciampaglia, Azadeh Nematzadeh, Filippo Menczer, and Alessandro Flammini. 2018. How algorithmic popularity bias hinders or promotes quality. Scientific reports 8, 1 (2018), 1-7.

[12] The Economist. 2020. Twitter's algorithm does not seem to silence conservatives. https://www.economist.com/graphic-detail/2020/08/01/twitters-algorithmdoes-not-seem-to-silence-conservatives

[13] Seth Flaxman, Sharad Goel, and Justin M Rao. 2016. Filter bubbles, echo chambers, and online news consumption. Public opinion quarterly 80, S1 (2016), 298-320.

[14] Santo Fortunato, Alessandro Flammini, Filippo Menczer, and Alessandro Vespignani. 2006. Topical interests and the mitigation of search engine bias. Proceedings of the national academy of sciences 103, 34 (2006), 12684-12689.

[15] Eduardo Hargreaves, Claudio Agosti, Daniel Menasché, Giovanni Neglia, Alexandre Reiffers-Masson, and Eitan Altman. 2018. Biases in the facebook news feed: a case study on the italian elections. In 2018 IEEE/ACM International Conference on Advances in Social Networks Analysis and Mining (ASONAM). IEEE, 806-812.

[16] Nathan O Hodas and Kristina Lerman. 2014. The simple rules of social contagion. Scientific reports 4 (2014), 4343.

[17] Adam Hughes and Stefan Wojcik. 2019 (accessed July 3, 2020). 10 facts about Americans and Twitter. https://www.pewresearch.org/fact-tank/2019/08/02/10facts-about-americans-andtwitter/

[18] Dietmar Jannach, Lukas Lerche, Iman Kamehkhosh, and Michael Jugovac. 2015. What recommenders recommend: an analysis of recommendation biases and possible countermeasures. User Modeling and User-Adapted Interaction 25, 5 (2015), 427-491.

[19] Juhi Kulshrestha, Motahhare Eslami, Johnnatan Messias, Muhammad Bilal Zafar, Saptarshi Ghosh, Krishna P Gummadi, and Karrie Karahalios. 2017. Quantifying search bias: Investigating sources of bias for political searches in social media. In Proceedings of the 2017 ACM Conference on Computer Supported Cooperative Work and Social Computing. 417-432.

[20] Kristina Lerman and Tad Hogg. 2014. Leveraging position bias to improve peer recommendation. PloS one 9, 6 (2014), e98914.

[21] Solomon Messing and Sean J Westwood. 2014. Selective exposure in the age of social media: Endorsements trump partisan source affiliation when selecting news online. Communication research 41, 8 (2014), 1042-1063.

[22] Dimitar Nikolov, Mounia Lalmas, Alessandro Flammini, and Filippo Menczer. 2019. Quantifying biases in online information exposure. Fournal of the Association for Information Science and Technology 70, 3 (2019), 218-229.

[23] Yoon-Joo Park and Alexander Tuzhilin. 2008. The long tail of recommender systems and how to leverage it. In Proceedings of the 2008 ACM conference on Recommender systems. 11-18.

[24] Matjaž Perc. 2014. The Matthew effect in empirical data. fournal of The Royal Society Interface 11, 98 (2014), 20140378.

[25] Ashwin Rao, Fred Morstatter, Minda Hu, Emily Chen, Keith Burghardt, Emilio Ferrara, and Kristina Lerman. 2020. Political Partisanship and Anti-Science Attitudes in Online Discussions about Covid-19. arXiv preprint arXiv:2011.08498 (2020). 
[26] Ronald E Robertson, Shan Jiang, Kenneth Joseph, Lisa Friedland, David Lazer and Christo Wilson. 2018. Auditing partisan audience bias within google search. Proceedings of the ACM on Human-Computer Interaction 2, CSCW (2018), 1-22.

[27] Manuel Gomez Rodriguez, Krishna Gummadi, and Bernhard Schoelkopf. 2014. Quantifying information overload in social media and its impact on social contagions. In Eighth International AAAI Conference on Weblogs and Social Media.

[28] Matthew J Salganik, Peter Sheridan Dodds, and Duncan J Watts. 2006. Experimental study of inequality and unpredictability in an artificial cultural market. Science 311, 5762 (2006), 854-856.

[29] Christian Sandvig, Kevin Hamilton, Karrie Karahalios, and Cedric Langbort. 2014 Auditing algorithms: Research methods for detecting discrimination on internet platforms. Data and discrimination: converting critical concerns into productive inquiry 22 (2014), 4349-4357.
[30] Clay Shirky. 2011. The political power of social media: Technology, the public sphere, and political change. Foreign affairs (2011), 28-41.

[31] Jessica Su, Aneesh Sharma, and Sharad Goel. 2016. The effect of recommendations on network structure. In Proceedings of the 25th international conference on World Wide Web. International World Wide Web Conferences Steering Committee, $1157-1167$.

[32] Twitter. 2020. About your Twitter timeline. https://help.twitter.com/en/usingtwitter/twitter-timeline

[33] Siqi Wu, Marian-Andrei Rizoiu, and Lexing Xie. 2020. Variation across Scales: Measurement Fidelity under Twitter Data Sampling. In Proceedings of the International AAAI Conference on Web and Social Media, Vol. 14. 715-725.

[34] JungAe Yang. 2016. Effects of popularity-based news recommendations ("mostviewed") on users' exposure to online news. Media Psychology 19, 2 (2016), 243-271 